\title{
El omeprazol antes de la endoscopía mejora el sangrado de las úlceras pépticas
}

\author{
Omeprazol before endoscopy improves bleeding in patients with peptic ulcer
}

Lau, J. y col. N Engl J Med. 2007, 356: 1631-40.

\section{Objetivo}

Evaluar el efecto del omeprazol administrado previamente a la endoscopía digestiva alta sobre la necesidad de tratamiento endoscópico hemostático en la hemorragia digestiva alta (HDA).

\section{Diseño}

Ensayo clínico controlado aleatorizado.

Lugar

Hong Kong, China.

\section{Pacientes}

638 pacientes consecutivos ingresados al departamento de emergencias con evidencia de HDA (melena o hematemesis con o sin hipotensión). Criterios de exclusión: shock refractario, edad menor de 18 años, embarazo, incapacidad para dar consentimiento, medicación previa con inhibidores de la bomba de protones (IBP) y/o aspirina en bajas dosis.

\section{Intervención}

Fueron aleatorizados 319 pacientes en cada rama (placebo vs. bolo endovenoso de $80 \mathrm{mg}$ de omeprazol seguido por una infusión continua de $8 \mathrm{mg} / \mathrm{h}$ ) hasta la realización de la endoscopía a la mañana siguiente. Las lesiones con sangrado activo y los vasos visibles fueron tratados endoscópicamente con infiltración de epinefrina y termocoagulación, manteniéndose la infusión de omeprazol durante $72 \mathrm{~h}$ y comenzándose luego con medicación oral. Ante resangrado se repetió la endoscopía y se derivó a cirugía a los pacientes con HDA no controlable endoscópicamente o con otro resangrado. Se pesquisó y trató el Helicobacter pylori. El seguimiento fue de 30 días.

Medición de resultados principales

Necesidad de terapia endoscópica analizada por intención de tratar* $^{*}$ Resultados secundarios: signos de sangrado, necesidad urgente de endoscopía, transfusiones o cirugía, estadía hospitalaria, tasas de resangrado y mortalidad por cualquier causa.

\section{Resultados principales}

Los asignados a omeprazol tuvieron menor requerimiento de tratamiento endoscópico. Ver tabla 1. Hubo menos sangrado activo por úlcera péptica en el grupo intervención que en el control $(6,4$ vs. $14,7 \%$; $p=0,01)$. En la rama que recibió omeprazol fueron más frecuentes las úlceras con fondo limpio (64,2 vs. $47,4 \% ; p=0,001$ ) y fue más corta la internación (mediana menor a tres días en el 60,5 vs. el $49,2 \%$ del grupo placebo; $p=0,005$ ) sin diferencias significativas en las tasas de resangrado, transfusiones, cirugía de emergencia y mortalidad.

Tabla: necesidad de tratamiento endoscópico en cada grupo.

\begin{tabular}{l|c|c|c}
\multirow{2}{*}{$\begin{array}{l}\text { Total de los } \\
\text { pacientes }\end{array}$} & $\begin{array}{c}\text { Rama asignada de } \\
\text { tratamiento }\end{array}$ & $\begin{array}{c}\text { Necesidad de terapia } \\
\text { endoscópica }\end{array}$ & RR (IC95\%) \\
\cline { 2 - 3 } & $\begin{array}{c}\text { Omeprazol } \\
(\mathrm{n}=314)\end{array}$ & $90(28,4 \%)$ & $0,67(0,51$ a 0,90$)$ \\
\hline Pacientes con & Placebo $(\mathrm{n}=190)$ & $70(36,8 \%)$ & \\
\cline { 2 - 3 } úlcera péptica & $\begin{array}{c}\text { Omeprazol } \\
(\mathrm{n}=187)\end{array}$ & $42(22,5 \%)$ & \\
\hline
\end{tabular}

\section{Conclusiones}

La infusión de omeprazol endovensoso en altas dosis horas antes de la primera endoscopía disminuyó los signos de sangrado, la necesidad de tratamiento endoscópico y la de internación.

Palabras clave: Hemorragia digestiva alta, omeprazol, tratamiento endoscópico. Keywords: upper gastrointestinal bleeding, omeprazole, endoscopic treatment. Fuentes de financiamiento: Instituto de Enfermedades Digestivas, Universidad China de Hong Kong. Conflictos de interés: Ios investigadores recibieron apoyo de AstraZeneca; Pfizer, Takeda and TAP Pharmaceutical Products y un grant de Pfizer y Glaxo-SmithKline, y se declararon sin conflictos de intereses.

\section{Comentario}

Es conocida la efectividad del tratamiento con IBP en la HDA, en particular en las lesiones pépticas sangrantes ${ }^{1,2}$. $\mathrm{Y}$, aunque se habían realizado ensayos con altas dosis de omeprazol endovenoso para evaluar las tasas de resangrado y la necesidad de cirugía, este protocolo es novedoso. La endoscopía de urgencia y la posibilidad de realizar tratamiento de lesiones gastroduodenales sangrantes por esta vía -de utilidad demostrada en ensayos controlados ${ }^{3,4}$ - no es común en la mayoría de las instituciones de nuestro país, por lo que, un tratamiento que disminuya el sangrado activo y la necesidad de terapéutica endoscópica puede ser de gran utilidad en la práctica diaria. Sin embargo, estos resultados no pueden generalizarse a todas las lesiones digestivas altas sangrantes, ya que fueron excluidos los individuos medicados crónicamente con aspirina en bajas dosis -una alta proporción de los casos con HDA y que puede implicar alteraciones en la formación de los coágulos hemostáticos.

La generalización de estos resultados debe relativizarse según cada zona geográfica y social y dependiendo de las causas de HDA, ya que donde el sangrado por várices esofágicas represente una alta proporción de casos, estas conclusiones podrían no tener el mismo valor práctico. Otro punto a considerar es la dosis de omeprazol indicada y su costo.

\section{Conclusiones del comentador}

El tratamiento de la HDA con IBP debería ser iniciado lo antes posible, en especial si no se dispone de endoscopía de urgencia.

Axel Beskow [ Especialista en Cirugía, Servicio de Cirugía General, Hospital Italiano de Buenos Aires axel.beskow@ hospitalitaliano.org.ar ]

Beskow A. La administración de omeprazol previo a la endoscopía acelera la resolución del sangrado de las úlceras pépticas. Evid. actual. práct. ambul; 10(5):139. Sep-Oct.2007. Comentado de: Lau, J, et al.Omeprazol before Endoscopy in Patients with Gastrointestinal Bleeding. N Engl J Med. 2007, 356 (16): 1631-40. PMID: 17442905

\section{Referencias}

1. Leontiadis GI, et al. Proton pump inhibitor treatment for acute peptic ulcer bleeding. Cochrane Database Syst Rev 2006;1:CD002094-CD002094.

2. Bardou M, et al. Meta-analysis: proton-pump inhibition in high-risk patients with acute peptic ulcer bleeding. Aliment Pharmacol Ther 2005;21:677-686.

3. Palmer K. Non-variceal upper gastrointestinal haemorrhage: guidelines. Gut 2002;51: Suppl 4:1-6.

4. Barkun A, et al. Consensus recommendations for managing patients with nonvariceal upper gastrointestinal bleeding. Ann Intern Med 2003;139:843-857. 